Service social

\title{
Politique sociale, politique partisane et profession
}

\section{Gérald Doré et Lorraine Gaudreau}

Volume 38, numéro 2-3, 1989

Les politiques sociales

URI : https://id.erudit.org/iderudit/706440ar

DOI : https://doi.org/10.7202/706440ar

Aller au sommaire du numéro

Éditeur(s)

École de service social de l'Université Laval

ISSN

1708-1734 (numérique)

Découvrir la revue

Citer cet article

Doré, G. \& Gaudreau, L. (1989). Politique sociale, politique partisane et profession. Service social, 38(2-3), 246-262. https://doi.org/10.7202/706440ar

\section{Résumé de l'article}

Les politiques sociales s'élaborent dans un processus complexe comportant plusieurs phases ou moments en interaction, dont celui de la prestation dans lequel interviennent les professionnels/le/s et technicien/ne/s des services sociaux. À l'aide de quelques publications récentes, cet article cherche à savoir si ceux-ci se confinent au moment qui leur est fonction-nellement assigné, ou s'ils interviennent également dans les autres phases qui appartiennent au champ de l'action politique. d'utilisation que vous pouvez consulter en ligne.

https://apropos.erudit.org/fr/usagers/politique-dutilisation/ 
DORÉ, Gérald, professeur à l'École de service social, Université Laval.

GAUDREAU, Lorraine, étudiante au doctorat à l'École de service social, Université Laval.

\section{Politique sociale, politique partisane et profession}

\section{Gérald Doré Lorraine Gaudreau}

Depuis Piven et Cloward (1971) aux États-Unis, Panitch (1977) au Canada, Pelletier et Vaillancourt (1974-1978 et Vaillancourt, 1988) au Québec, on peut difficilement continuer à voir la politique sociale comme la manifestation concrète d'un humanisme en voie de résorber les "abus du capitalisme ", ou encore comme le terrain neutre du développement " rationnel " de la satisfaction des besoins. On sait maintenant qu'il n'y a d'explication de la politique sociale que dans la reconstruction de son rapport à l'économique et au politique dans une société donnée. On a aussi pris acte qu'elle ne se développe pas nécessairement suivant la courbe ascendante $d^{\prime} u n$ évolutionnisme progressif. Son articulation aux mutations contradictoires dans les conjonctures économiques et politiques entraîne aussi bien des phases de régression que des phases de progression.

La politique sociale remplit une fonction de régulation politique. Elle contribue à désamorcer l'agitation sociale, à mobiliser des clientèles électorales, à intégrer de nouveaux leaders politiques. La mise en œuvre des politiques sociales dans une société concrète, située et datée, est liée à la division structurelle du pouvoir politique entre les différents paliers de l'État, de même qu'aux conflits d'intérêt à l'intérieur des classes dominantes (Doré, 1978 : 6-7). De plus, comme le rappelle Vaillancourt (1983: 75) :

Hier comme aujourd'hui et demain, le poids et la qualité des stratégies et luttes du mouvement ouvrier et populaire ainsi que le 
rapport de force plus ou moins avantageux que le camp du mouvement ouvrier et populaire réussit à développer dans sa résistance aux stratégies et luttes du camp du capital constituent, dans nos sociétés capitalistes, une véritable clé pour comprendre et changer le développement des politiques sociales.

\section{La sphère des rapports sociaux où s'élaborent les politiques sociales}

En fait, les politiques sociales s'élaborent dans un processus dialectique à l'intérieur d'une sphère de rapports sociaux. Les acteurs et actrices* en interaction dans cette sphère sont : les groupes d'intérêt constitués et les mouvements en émergence qui font PRESSION sur le système politique partisan; les partis qui assument la DÉLÉGATION d'intérêts dans ce système; la bureaucratie d'État à qui revient I'ADMINISTRATION, c'est-à-dire la transformation, en programmes d'action, des buts plus ou moins bien définis des politiciens; enfin, les différents corps d'emploi de professionnels et professionnelles et de techniciens et techniciennes affectés à la PRESTATION en argent ou en services qui découle des politiques adoptées. Ces différents moments du processus sont représentés dans la figure 1.

\section{Figure 1}

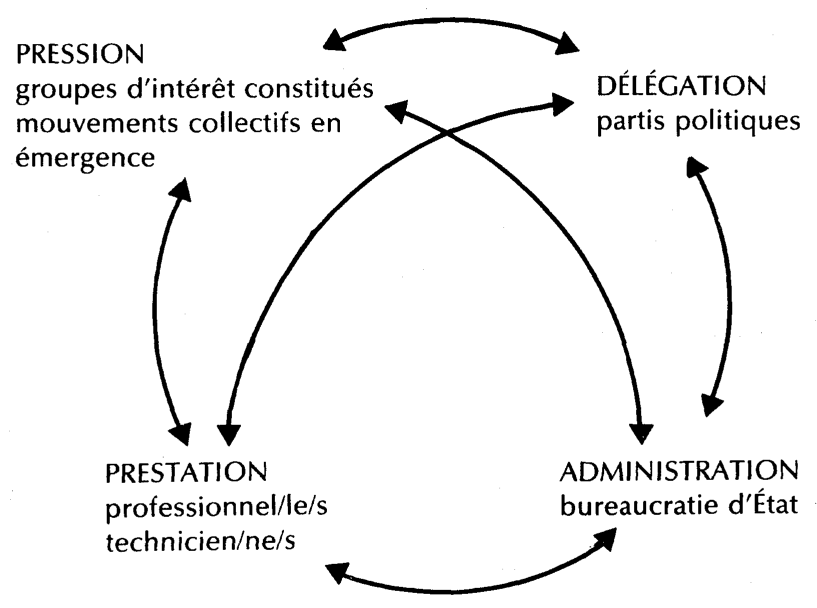


Des rapports complexes, et éventuellement contradictoires, s'établissent entre acteurs et actrices individuels et collectifs d'un même moment du processus, en même temps qu'entre acteurs et actrices de moments différents. Même si le processus peut théoriquement être activé de partout, un certain ordre tend à y prévaloir. L'émergence, le développement, le contenu et l'orientation de politiques sociales nouvelles, faisant plus de place aux droits collectifs et au partage des risques sociaux, y sont le produit de l'activité des mouvements sociaux axés sur le changement (pression) et des partis politiques qui leur sont liés (délégation), avant d'être institutionalisés dans l'activité administrative de l'État et la prestation de paiements de transfert ou de services. Le recul sur les acquis en matière de politique sociale émergera plutôt de l'activité de groupes d'intérêt déjà dominants (pression) et de partis politiques qui leur sont inféodés (délégation), avant d'être avalisé dans le fonctionnement quotidien des appareils d'État. La définition de ces moments du processus et des rapports sociaux qui les investissent appelle quelques clarifications.

\section{Pression}

En opposition aux groupes d'intérêt constitués qui bloquent, freinent ou font reculer les politiques sociales, une partie importante des acteurs et actrices qui les promeuvent. se retrouvent dans des organisations syndicales, populaires ou communautaires. Ils et elles exercent leur pouvoir dans ce que Gramsci appelle la société civile (Macciocchi, $1974: 164)^{1}$, à l'extérieur donc des partis politiques et dans les espaces que la démocratie leur laisse pour actualiser leurs intérêts. Reprenant ce concept de société civile, Touraine affirme que celle-ci « n'a d'existence autonome que si on reconnaît qu'elle repose sur des rapports sociaux fondamentaux, rapports qui résistent à la représentation politique en même temps qu'ils l'appellent " (Touraine, 1983 ; 133). Les hommes et les femmes qui agissent dans la société civile ne commencent pas à exister quand les politiciens et politiciennes prennent le relais de leurs revendications. Ils et elles ont une identité propre et un pouvoir à travers leurs organisations.

Comme le souligne Vaillancourt (1983 : 75-76) :

La montée de la conscience, des revendications, de l'unité et de la combativité du côté des diverses organisations du mouvement ouvrier et populaire oblige l'État capitaliste à faire des concessions plus avantageuses pour les classes populaires à travers les politiques sociales.

Ajoutons qu'historiquement, les luttes du champ des politiques sociales se sont installées en force dans les rapports sociaux de la 
société québécoise, au cours des années soixante, et que plusieurs études en rendent compte. Entre autres : Valois et Paquet, 1974; Pelletier et Vaillancourt, 1974-1978; Castells, 1975; Welch, 1975; Andrew, Blais et Des Rosiers, 1976; Collin et Godbout, 1977; McGraw, 1978; Doré, 1978; Doré et Plamondon, 1980; Doré et Mayer, 1981; Robert et Racicot, 1981; Arcand, 1981; Fortin et Roland, 1981; Roberge, 1981; Andrew, Bordeleau et Guimont, 1981; Hamel, 1983; Ventelou, 1983; René et Panet-Raymond, 1984; Poirier, 1986; Leboeuf, 1987.

\section{Délégation}

La présence ou l'absence sur la scène politique de partis qui portent la délégation des intérêts de chacune des classes sociales influence la nature même des politiques sociales. Le concept de délégation politique réfère à l'accès au système de la politique partisane, à la "société politique », pourrions-nous ajouter, pour nous situer dans le contexte plus large de la distinction effectuée par Gramsci entre société civile et société politique. Au moment de la pression, se manifeste "la demande que la société adresse au système ", alors que cette étape-ci met à l'ordre du jour la "délégation que (la société) donne aux sujets politiques d'agir dans le système, pour arriver à le gouverner »(Pizzorno, 1980 : 14).

L'accélération des luttes populaires sur des enjeux de politique sociale, depuis les années soixante, a correspondu à une phase au cours de laquelle se réalisait ce que Pizzorno appelle " I'acquisition d'un rôle même minime dans la société politique de la part de nouvelles masses de population ». Dans un tel contexte, "les partis font surface, soit de nouveaux partis, soit des partis déjà exisțants qui se transforment et amplifient leur activité ». L'effet de leur présence est alors "soit d'identifier et transmettre la nouvelle demande sous un mode plus efficace et pressant, mais aussi plus coordonnée et plus techniquement recevable, soit de recueillir de manière unifiée lá délégation des nouvelles masses »(Pizzorno, $1980: 20-21)$.

Au début des années soixante, le Parti Libéral a assumé une certaine "délégation " du syndicalisme pour la mise à jour du droit du travail et le développement de la politique sociale. De sa fondation à la fin de son premier mandat, le Parti Québécois s'est présenté et a été vu par plusieurs comme le dépositaire du développement des politiques sociales. II faudra le second mandat, avec son option résolument néo-conservatrice, pour que se rompe ce pacte tacite de la représentation entre le Parti Québécois et les mouvements axés sur I'amélioration des conditions de vie des classes populaires. La levée de l'illusion péquiste a rendu manifeste l'absence sur la scène 
politique québécoise d'un parti qui défende les intérêts des classes populaires. L'existence d'un tel parti est essentielle dans le processus dialectique qui préside à l'élaboration des politiques sociales. Sinon, il devient impossible d'ouvrir une brèche dans le mur politique qui empêche l'actualisation des revendications du mouvement populaire. Un projet de société ne peut s'actualiser qu'en investissant le champ de la politique partisane, dans " une volonté explicite de conquérir le pouvoir d'État » (Chancel, $1981: 13$ ).

\section{Administration}

$C^{\prime}$ est dans l'administration publique, à travers la mission sociale de l'État, que s'institutionnalisent les politiques sociales. Elles s'y distribuent en huit grands domaines d'intervention, chapeautés par un objectif d'harmonisation de l'ensemble. Ces domaines sont la sécurité sociale, la sécurité du revenu, la santé, les services sociaux, I'habitation, l'environnement, le travail et la main-d'œuvre, l'éducation.

Les acteurs et actrices de ce moment du processus sont ceux et celles que Merton appelait les intellectuels dans la bureaucratie (Merton, 1965) et qu'on appelle plus volontiers aujourd'hui les technocrates. "Le client de l'intellectuel bureaucrate est, de façon directe ou indirecte, un politicien qui veut transformer en programme d'action des buts plus ou moins bien définis. Les exigences de ce client peuvent se classer en plusieurs types ». Merton les énumère en commençant par ceux où le technocrate se voit octroyer le plus de liberté et en concluant par la considération suivante qui rend bien compte de ce qui caractérise ce moment du processus :

II est plus courant pour l'intellectuel d'être appelé à donner des précisions sur des orientations politiques déjà déterminées par les politiciens. On lui demande de montrer quelles sont les raisons de préférer l'un des termes de l'alternative envisagée ou quels points doivent entrer en ligne de compte dans l'exécution d'une politique déterminée (...) II envisage seulement les conséquences et les modes de réalisation propres aux décisions qu'on lui a soumises (Merton, 1965 : 362-364).

\section{Prestation}

Au moment de la prestation, le client n'est plus le politicien, mais le bénéficiaire ou l'usager d'allocations ou de services qui découlent de l'adoption et de l'implantation des politiques. Les acteurs et actrices de ce moment du processus sont les professionnels et professionnelles et les techniciens et techniciennes spécialisés dans 
le type particulier de prestation qui constitue l'extrant du processus. Le rôle qui confère l'identité professionnelle s'actualise dans une relation directe avec le public.

Dans le cas des professionnels et professionnelles et des techniciens et techniciennes des services sociaux, qui interviennent sous divers titres d'emploi, dont les plus courants sont ceux d'agent ou agente de relations humaines, technicien ou technicienne en assistance sociale, organisateur ou organisatrice communautaire, travailleur ou travailleuse communautaire, la relation avec le public est une relation d'aide qui se réalise par la mobilisation des capacités autonomes des clients (individus, couples, familles, groupes et collectivités) et l'utilisation des ressources communautaires rendues disponibles par l'ensemble des politiques sociales en vigueur. La place qui est fonctionnellement assignée aux professionnels et professionnelles et aux techniciens et techniciennes des services sociaux dans la sphère d'élaboration des politiques sociales se situe ici, à ce moment de la PRESTATION. La question qui nous intéresse, et qui fait écho au troisième volet du titre de cet article, est précisément de savoir s'ils se confinent à cette phase-ci du processus, ou s'ils interviennent également dans les autres moments qui sont ceux de la PRESSION, de la DÉLÉGATION et de I'ADMINISTRATION. Nous tenterons de répondre à cette question à l'aide de quelques publications récentes sur le sujet.

\section{La place de la profession}

Mahaffey et Hanks ont dirigé, en 1982, la publication, aux ÉtatsUnis, d'un ouvrage central par rapport à la question qui nous occupe ici. Vingt auteurs et auteures y ont participé à travers quinze contributions. Les textes récents sur le sujet y font fréquemment référence. Mahaffey et Hanks (1982: vi) qualifient eux-mêmes leur livre d'unique :

II n'est pas surprenant que les travailleuses sociales et travailleurs sociaux, avec leur intérêt marqué pour les programmes communautaires, les relations inter-groupes et l'action sociale, soient souvent fortement impliqués dans des enjeux et des campagnes politiques. Ce qui surprend, c'est que la littérature de la profession offre si peu de contenu qui mette en rapport les valeurs, objectifs et habiletés du service social avec le processus politique. Relativement peu d'articles ont traité un aspect ou l'autre du sujet. Ce livre est le premier à le faire.

À partir d'expériences concrètes, cet ouvrage cherche à répondre à un vaste éventail de questions : quels sont les précédents 
historiques au niveau de l'action politique des travailleuses sociales et travailleurs sociaux? quels sont les principes qui sous-tendent la reconnaissance d'une composante politique dans la pratique du service social ? quelles habiletés doit développer la travailleuse sociale ou le travailleur social pour être efficace dans son rôle de " critique " des politiques sociales, ou encore comme candidate ou candidat ? comment le fait d'être une politicienne ou un politicien élu favorise-t-il l'atteinte des objectifs du service social ?

Le livre affirme la contribution spécifique des travailleuses sociales et travailleurs sociaux dans le champ de la politique partisane et reconnaît que celle-ci peut favoriser l'atteinte des objectifs de la profession :

En tant que travailleuse sociale ou travailleur social, j'ai une conception des enjeux qui tend à être humaniste plutôt que légaliste...

... la méthode traditionnelle de résolution de problème en situation thérapeutique... se transpose facilement dans le champ politique.

Ce livre est un premier pas pour expliquer comment le savoir, l'expérience et les habiletés du service social s'appliquent au processus politique et comment la participation politique peut aider les travailleuses sociales et les travailleurs sociaux à remplir leurs responsabilités professionnelles (Mahaffey et Hanks, 1982 : vi et vii).

La place des travailleuses sociales et travailleurs sociaux dans les institutions politiques $y$ est, dès le départ, située au niveau du processus d'élaboration des politiques sociales, dans le sens d'un accroissement de leur participation dans les institutions politiques qui sont responsables de cette élaboration (Mahaffey et Hanks, 1982 : v). Et même si l'ouvrage met l'accent sur le service social comme profession, il est présenté comme pouvant être "également utile aux membres des autres professions d'aide dont la pratique est aussi fortement tributaire des politiques sociales " (Brieland, 1982 : 2).

Deux grands types d'implication politique sont identifiés par Mahaffey et Hanks : les activités "souterraines " et les postes de leadership. Militer à l'intérieur d'un parti comme travailleuse ou travailleur d'élection, conseillère ou conseiller, ou encore agente ou agent politique, sont des exemples d'activités décrites comme cachées. Être candidat officiel, candidate officielle lors d'élections, occuper la présidence dans une coalition ou faire du lobbying à temps plein pour une association professionnelle représentent des rôles de leaders. Certaines de ces activités politiques demandent d'ailleurs à être précisées, puisqu'on ne trouve pas leur pendant dans la réalité québécoise actuelle. 


\section{Le moment de la pression : lobbying et coalition}

À partir de la constatation faite par la National Association of Social Workers (N.A.S.W.) lors de sa fondation, en 1955, du lien direct entre l'activité quotidienne des travailleuses sociales et travailleurs sociaux et la politique sociale, s'est développée l'idée de la nécessité de s'impliquer politiquement, ce qui a eu pour effet de faire de l'activité politique "une obligation pour la travailleuse sociale professionnelle ou le travailleur social professionnel, de même que pour l'association professionnelle (Brieland, $1982: 4$ ).

Dans la foulée de cette orientation, s'est développé, au début des années soixante-dix, un moyen considéré comme privilégié pour influencer l'élaboration des politiques sociales, un moyen qui s'insère dans la phase de la pression : le lobbying. L'association déménagea alors son siège social à Washington (D.C.), et engagea des travailleuses sociales et travailleurs sociaux, pour appliquer cette stratégie déjà bien connue des milieux politiques, dans le but d'infléchir les projets de politique sociale dans le sens des intérêts de la majorité de la clientèle des services sociaux et des bénéficiaires des programmes sociaux. Cette activité politique de pression recourt à diverses tactiques : "lutte, confrontation, négociation, compromis, formation de coalitions et exercice d'un pouvoir... » (Brieland, 1982:6). C'est ainsi, par exemple, que la Coalition du Wyoming a obtenu l'appui de plus de trente organismes pour l'obtention d'un programme de supplément alimentaire pour les femmes et les enfants.

Le livre de Mahaffey et Hanks nous présente d'ailleurs la N.A.S.W. davantage sous l'angle de la promotion des droits de la clientèle des services sociaux que sous celui de la légitimation du statut de ses membres. L'association a en effet créé deux structures pour favoriser l'action politique de ses adhérents. L'Educational Legislative Action Network (E.L.A.N.) a été fondé en 1971, pour "poursuivre les objectifs législatifs de l'association par le lobbying". Le Political Action for Candidate Election (P.A.C.E.) a pour sa part été créé en 1975, pour amasser des fonds en vue de favoriser la campagne électorale de candidats et candidates en accord avec le programme de I'association. À cet égard, Brieland (1982: 10) souligne que "la collecte de fonds est une fonction critique dans n'importe quel comité d'action politique, mais que c'est une tâche bien difficile dans le milieu du service social où on n'a pas l'habitude d'appuyer financièrement les candidats aux élections. » 


\section{Le moment de la délégation : candidature de travailleuses sociales et travailleurs sociaux aux élections}

"Se présenter et gagner, écrit Brieland (1982: 9) est de toute évidence le moyen le plus efficace de s'assurer que les questions relatives au bien-être social soient représentées dans les assemblées législatives. " Et c'est un point de vue partagé par cinq membres de la profession qui siègent ou ont siégé à l'Assemblée législative de la Colombie-Britannique, dans les rangs du Nouveau Parti Démocratique (N.P.D.), et dont Marschall et Strandberg ont recueilli les témoignages dans un article récent (1988).

Partant de la constatation que, depuis les années soixante, de plus en plus de travailleuses sociales et travailleurs sociaux s'impliquent politiquement, pour atteindre leur visée de changement social, les auteures cherchent à cerner la nature de cet engagement, en portant une attention spéciale aux compromis que peut exiger la politique partisane et à la contribution spécifique du service social dans le champ de la société politique. Avant d'en arriver aux entrevues, elles font état du contenu de deux débats historicoidéologiques, au sein du service social, qui colorent l'implication politique actuelle de ses commettants. Le premier a trait à l'évaluation de la pertinence d'un modèle comme moteur d'amélioration des conditions de vie de la clientèle des services sociaux: est-ce le modèle d'intervention individuelle ou collective? Le second est centré sur l'évaluation de l'idéologie d'action politique la plus efficace pour transformer la société : le réformisme ou le service social " radical»? Pour certains travailleurs sociaux et travailleuses sociales s'ajoute la question de l'éthique professionnelle, du défi éthique qui est «qualitativement et quantitativement plus important pour ceux et celles qui sont investis d'une fonction politique" (Marshall et Strandberg, $1988: 112$ ).

La parole politique des politiciens et politiciennes interrogés place pourtant les conflits et les compromis beaucoup plus dans la pratique de la profession, à l'intérieur des établissements, dans le moment de la prestation donc, que dans la politique partisane. "Je souhaiterais, déclare la travailleuse sociale et députée Rosemary Brown, que (les travailleuses sociales et travailleurs sociaux) se rangent du côté du client et se sentent partie prenante dans la lutte du client pour l'amélioration de sa qualité de vie " (Marshall et Strandberg, 1988 : 113).

La formation universitaire qui n'incite ni à une analyse structurelle de la société, ni à une action politique, ni à une alliance de classes est critiquée : 
... là où le service social m'a laissée interloquée, affirme Darlene Mazari, c'est dans son incapacité à focaliser sur l'analyse politique. II y avait un message caché, ajoute Rosemary Brown, qui disait de ne pas mêler la politique avec le service social.

À cause de la méthode thérapeutique à partir de laquelle le service social a évolué, renchérit Darlene Mazari, il ne te pousse pas vers les analyses de classes et de groupes socio-économiques en lutte les uns contre les autres. Ce n'est pas quelque chose que le modèle thérapeutique a encouragé ou développé (Marshall et Strandberg, 1988 : 113).

Des interventions nous suggèrent la nécessité d'une alliance de classes. La travailleuse sociale et députée Margaret Mitchell souligne notamment les contradictions que cette alliance peut soulever :

Je voulais participer au changement social et aider les gens à réaliser ce changement.

J'ai toujours été très sensible au fait que la plupart des travailleuses sociales, y compris moi-même, avaient une bonne éducation... et nous avons eu toute cette formation pour sortir ensuite et aller travailler en milieu ouvrier (Marshall et Strandberg, 1988 : 113).

D'après les politiciens et politiciennes interrogés, l'implication politique des travailleuses sociales et travailleurs sociaux se réalise : 1) dans le développement de leur conscience socio-politique, qui devrait les amener entre autres à questionner l'État sur l'orientation qu'il donne aux politiques sociales, et à agir dans le champ de la société civile avec les citoyens des communautés qu'ils et elles desservent; 2) par l'implication dans leur association professionnelle, à partir d'une conception plus politique de cette implication, qui amène à la responsabilisation sociale face aux injustices produites par le système; 3) par l'action sociale devant amener des transformations dans la société, ce qui va jusqu'à impliquer que dans le quotidien du travail en établissement, on " subordonne les intérêts de l'employeur à ceux du client »; 4) par le biais, enfin, de la politique partisane, dans le sens où celle-ci est, selon Emery Barnes, " un moyen de réaliser les objectifs du service social " (Marshall et Strandberg, 1988 : 114).

Le social et la politique, et par conséquent la politique sociale, sont au cœur de l'implication des travailleuses sociales et travailleurs sociaux interrogés qui conviennent que « la politique et les décisions politiques sont d'une importance vitale, parce que la politique a trait aux enjeux de la qualité de vie et aux interactions et relations humaines" (Marshall et Strandberg, 1988 : 114).

Une deuxième partie de l'article rend compte de la contribution spécifique du service social à la politique partisane. Quatre dimensions sont présentées: les valeurs propres au service social, par exemple l'empathie; la capacité d'analyser les causes des problèmes 
sociaux; la capacité de se voir dans un processus, à l'intérieur de l'ensemble des rapports sociaux; les acquis techniques tels que l'écoute, les techniques d'animation, etc.

La conclusion est claire. Le problème pour les travailleuses sociales et les travailleurs sociaux n'est pas de faire de la politique mais bien de ne pas en faire suffisamment, surtout dans l'arène politique même. "Les travailleurs sociaux ne peuvent s'appeler eux-mêmes travailleurs sociaux, affirme le travailleur social, ex-premier ministre de la Colombie-Britannique et député à la Chambre des Communes Dave Barrett, s'ils ne voient pas qu'ils ont une responsabilité morale, professionnelle et éthique à être impliqués dans le monde de la politique, avec un petit « $p$ » comme avec un grand « $P$ », qui les entoure " (Marshall et Strandberg, $1988: 115$ ).

II n'aura échappé à l'attention de personne que les travailleuses sociales et travailleurs sociaux politiciens interrogés sont tous d'allégeance socialiste démocratique ${ }^{2}$. II n'est certes pas trop hasardeux de présumer que les propos de la travailleuse sociale et ex-ministre libérale de la Santé et des Services sociaux du Québec, Thérèse Lavoie-Roux, sur le même sujet, auraient pris une couleur différente. Le passage de l'organisatrice communautaire et ex-députée péquiste Pauline Marois au ministère de la Main-d'œuvre et de la Sécurité du revenu nous rappelle aussi qu'il ne suffit pas que la politique partisane soit investie par des travailleuses sociales ou des travailleurs sociaux pour que les politiques sociales favorisent le mieux-être de la clientèle des services sociaux ${ }^{3}$. Le débat sur l'idéologie d'action politique la plus efficace pour transformer la société, auquel Marshall et Strandberg (1988: 112) font allusion, reste un débat de premier plan.

\section{Idéologie et politique}

Rino J. Patti développe ce thème des idéologies sous-jacentes à I'action politique des travailleuses sociales et travailleurs sociaux. II définit I'action politique comme :

... les efforts délibérés d'individus et de groupes pour influencer les décisions politiques en participant aux élections, en identifiant les problèmes qui requièrent l'attention du gouvernement, en informant les décideurs politiques des alternatives au niveau de l'action, et des conséquences prévisibles, en influençant leurs choix à cet égard, et en plaidant pour le changement (Patti, 1983 : 96).

II évalue que, dans le sens de cette définition, l'action politique des travailleuses sociales et travailleurs sociaux en vue d'influencer les politiques sociales, suit présentement une courbe ascendante, aux 
États-Unis. Les faits suivants confirmés par une enquête menée auprès de deux cent quatre-vingt-neuf travailleuses sociales et travailleurs sociaux du Michigan expliquent, selon Patti, cette remontée : la dure réalité de la crise économique avec sa kyrielle de coupures qui affectent directement la clientèle des services sociaux; le désengagement de la politique reaganienne dans le domaine de la politique sociale, la perte donc de droits acquis au niveau des services de santé, des services sociaux et des programmes sociaux; le transfert administratif des programmes sociaux des états vers les municipalités.

Patti identifie deux grands courants idéologiques dont les travailleuses sociales et travailleurs sociaux sont tributaires : la "réforme sociale » et le« service social radical ». Le courant réformiste est, d'après lui, celui qui rassemble le plus d'adhérents. II se caractérise par un malaise à l'égard des institutions politiques et la conviction que l'État peut réduire la portée des problèmes sociaux. Les stratégies d'action utilisées sont de type traditionnel : coalition, éducation et compromis. On y voit le processus de décision politique qui compose le champ de la politique sociale comme "le résultat du jeu d'intérêts multiples et diversifiés, plutôt que des diktats de quelques élites économiques et politiques»(Patti, 1983 : 99).

Le courant radical implique la reconnaissance des institutions sociales comme agents d'exploitation et d'oppression. Les tenants de cette idéologie évaluent que les réformistes ont intégré les valeurs et la visée du système capitaliste. Le service social est vu comme moteur de reproduction des inégalités; donc s'y intégrer équivaut à refuser d'investir dans la lutte pour transformer radicalement la société. Les stratégies traditionnelles de jeux d'influences politiques sont rejetées.

L'idéologie du service social radical suggère plutôt une approche globale de l'action politique, avec l'objectif de transformer les institutions sociales par l'action collective de masse.

Les travailleuses sociales et travailleurs sociaux sont vus comme pouvant jouer un rôle instrumental dans cette lutte, mais seulement dans la mesure où ils se joignent à d'autres mouvements impliquant travailleurs, clients et militants sociaux (Patti, 1983 : 99 et 100).

Patti souligne que ces deux idéologies sont des pôles entre lesquels et au-delà desquels se situent les travailleuses sociales et travailleurs sociaux.

\section{Conclusion}

Dans les écrits parcourus, l'action politique des travailleuses sociales et travailleurs sociaux est directement reliée à l'analyse de l'effet des politiques sociales sur les conditions de vie de leur 
clientèle, le mieux-être de celle-ci étant une des visées de la profession du service social. L'action politique dont il s'agit se réalise à deux moments du processus d'élaboration des politiques sociales : celui de la pression sur les pouvoirs (par le lobbying et l'implication avec les communautés) et celui de la politique partisane (par le travail d'élection et les candidatures aux élections).

On trouve peu ou pas de contenu qui nous permette de cerner la place des travailleuses sociales et travailleurs sociaux de formation dans les paliers supérieurs de l'administration publique. Secret bien gardé ? C'est pourtant un moment important de l'élaboration des politiques sociales. Sont aussi passées sous silence les contributions des chercheurs et chercheuses qui diffusent des analyses critiques sur les politiques sociales. Qu'il s'agisse de chercheurs et chercheuses professionnels, de professeurs et professeures en service social ou de militants et militantes de groupes populaires ou de syndicats, ils et elles participent à la diffusion d'une contre-idéologie et alimentent les mouvements sociaux dans l'élaboration de leurs plans d'action. Outre les travaux du politicologue Yves Vaillancourt et de l'économiste Michel Pelletier déjà cités, mentionnons, à titre d'exemples, la recherche du collectif EZOP-Québec sur la pauvreté urbaine à Québec (AA. VV., 1981), I'analyse de la réorientation des politiques sociales dans la conjoncture de crise de la fin des années soixante-dix, publiée par I'Association pour la défense des droits sociaux du Québec métropolitain (A.D.D.S.Q.M.) et un collectif de recherche (A.D.D.S.Q.M., 1983), la série des cahiers du Groupe de recherche en action populaire (G.R.A.P.) sur les luttes relatives à l'aide sociale, au logement et à la protection des consommateurs (Fortin, 1985) et le récent ouvrage du sociologue Denis Fortin sur le double régime d'assistance sociale au Canada (Fortin, 1988).

Que la présence dans la politique partisane de travailleuses sociales et travailleurs sociaux ne soit pas garante du mieux-être de la clientèle des services sociaux et des bénéficiaires des programmes sociaux n'empêche pas de constater l'importance de déboucher sur la politique partisane, pour vraiment exercer un pouvoir dans le champ de la politique sociale. Au Québec comme ailleurs, des intervenantes et intervenants sociaux l'ont compris depuis longtemps, mais le manque de recherches à ce sujet contribue à y entretenir le préjugé, dénoncé par la députée Brown, qu'il ne faut pas "mêler la politique avec le service social ".

À cet égard, l'importance des valeurs véhiculées dans la profession et des objectifs de formation dans les écoles de service social est affirmée. Au moins deux des textes cités font aussi état des compromis et des conflits vécus dans les lieux de travail, c'est-à-dire dans le moment même de la prestation. La question de l'alliance qui 
peut s'établir entre les intervenantes et intervenants sociaux et la base populaire qui constitue leur clientèle majoritaire $n^{\prime}$ est traitée que sous forme d'allusion, dans la description de l'idéologie du service social radical, et la reconnaissance par la députée Mitchell des contradictions de classe que peuvent porter les travailleuses sociales et travailleurs sociaux. L'importance de travailler POUR le mieux-être de la clientèle est donc davantage affirmée que celle de travailler AVEC elle.

Toutes ces considérations nous font bien voir l'importance de traverser la sphère d'élaboration des politiques sociales, dans chacun des moments définis, de questions qui lui confèrent une portée et un sens ouverts sur le changement, dans une société concrète, bien située et datée. Autrement, elle risque de n'être qu'un cadre de référence formel, un schéma de rationalisation après coup de pratiques bien établies et prises pour acquises.

Nous conclurons donc par une nouvelle illustration de la sphère des rapports sociaux qui président à l'élaboration des politiques sociales, enrichie des questions que nous suggèrent notre réflexion et notre exploration des publications sur le sujet.

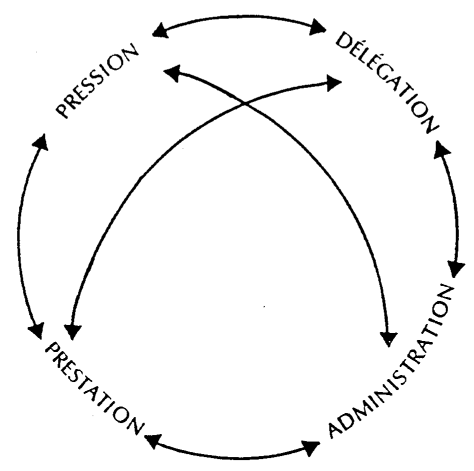

\section{Variables d'analyse}

Quelles sont les forces sociales qui agissent dans le champ de la société civile sur des enjeux de politique sociale?

Quelles sont les idéologies, visées et valeurs portées par les acteurs et les actrices en présence?

Quels sont les objectifs au niveau de l'action et de l'organisation des groupes impliqués?

\section{Variables d'analyse}

Les partis politiques en présence sont porteurs des intérêts de quelle/s classe/s sociale/s ?

Quel est leur programme, I'idéologie principale qui le sous-tend, leur conception de la mission sociale de l'État, etc. ? 
Dans quel type d'organisation se rassemblent les acteurs et les actrices en présence : fonctionnement, structures, stratégies et tactiques? Quelle est la situation, l'option de classe des acteurs et actrices impliqués?

Quels types de rapports sociaux sont établis entre les acteurs et actrices de la petite bourgeoisie et ceux et celles des classes populaires?

Etc.

Champ de la société civile : les pratiques des groupes d'intérêt constitués et les luttes des mouvements sociaux pour faire pression sur les pouvoirs.

Champ de la pratique professionnelle : la relation directe avec le public usager des services ou bénéficiaire des paiements.

\section{Variable d'analyse}

Quels sont les contradictions et les conflits vécus par les intervenantes et intervenants dans les établissements où se réalise la prestation?
Jusqu'à quel point les acteurs et actrices de ces partis sont en accord avec les intérêts défendus dans l'exercice du pouvoir ?

Le parti au pouvoir représente-t-il vraiment les intérêts à partir desquels il a été élu?

Etc.

Champ de la société politique : le parlement, le gouvernement, les partis politiques qui portent la délégation des intérêts des classes et couches sociales.

Champ de l'administration publique : la mission sociale de l'État dans les huit domaines d'intervention où sont mises en œuvre les politiques sociales.

\section{Variable d'analyse}

Quelle analyse de la société, et donc quelle orientation au niveau des politiques sociales, est portée par les concepteurs et conceptrices de ces politiques dans la bureaucratie d'État?

\section{Notes}

* À la demande des auteurs, les génériques masculin et féminin sont utilisés dans I'article.

${ }^{1}$ "On peut, écrit GramsCl,... distinguer deux grands " niveaux» dans la superstructure, celui qu'on peut désigner comme "société civile ", c'est-àdire l'ensemble des organismes, habituellement appelés "internes et privés", et celui de la "société politique ou État", correspondant respectivement à la fonction $\mathrm{d}^{\prime}$ ' " hégémonie " que le groupe dirigeant exerce sur l'ensemble du corps social et à celle de "domination directe " ou de commandement, qui s'exprime à travers l'État et le pouvoir "juridique ". Cité dans MACCIOCCHI, M.-A., Pour Gramsci, Paris, Seuil, Coll. "Points", 1974, 164.

${ }^{2}$ Au début de 1989, il était déjà sérieusement question de la candidature de la travailleuse sociale et députée du Yukon, Audrey MCLAUGHLIN, à la direction du N.P.D. fédéral. Celle-ci faisait alors elle-même le lien entre sa pratique du service social et son engagement en politique. "Son travail en service social à Toronto, rapportait le journaliste qui l'avait interviewée, l'amène cependant à croire que les gens pauvres sont trop souvent victimes du système et que 
l'action politique peut influencer le cours des choses. "Du moins un peu ", ajoutait-il en la citant. " Je ne dis pas que le N.P.D. a des réponses parfaites pour tout", confirmait-elle. "Mais la philosophie de son parti, concluait le journaliste, est à son avis plus soucieuse de justice sociale que les autres. " Voir : NOREAU, P.P., "Une femme à la tête du N.P.D... MCLAUGHLIN pourrait surprendre ", Le Soleil, Québec, 11 mars 1989, B-11 et B-12.

${ }^{3}$ Le gouvernement péquiste dans lequel a siégé la ministre Pauline MAross, a jeté les bases des changements que les libéraux font aujourd'hui subir à la Loi de l'aide sociale et que dénonce la Corporation professionnelle des travailleurs sociaux du Québec, après bien d'autres groupes socio-économiques. Voir : Boullle-DrouIN, O., "Une réforme qui nous coûtera socialement très cher ", Le Soleil, Québec, 19 mars 1989, B-6.

\section{Références bibliographiques}

AA. VV., Une ville à vendre, Montréal, Saint-Martin, 1981.

ANDReW, C., A. Blais et R. Des Rosiers, Les élites politiques, les bas-salariés et la politique du logement à Hull. Ottawa, Éditions de I'Université d'Ottawa, 1976.

ANDREW, C., S. Bordeleau et A. Guimont, L'urbanisation : une affaire. L'appropriation du sol et l'État local dans l'Outaouais québécois, Ottawa, Éditions de l'Université d'Ottawa, 1981.

ARCAND, M., La participation des locataires à la gestion des H.L.M. de Québec, de 1971 à 1978, thèse de maîtrise en service social, Québec, Université Laval, 1981.

Association pour la défense des droits sociaux du Québec métropolitain (A.D.D.S.Q.M.) et un collectif de recherche. Revenu minimum garantiou pauvreté maximum garantie, Québec, A.D.D.S.Q.M., 1983.

BRIELAND, D., "Introduction" dans MAHAFFeY, M. et J.W. HANKS (sous la direction de), Practical Politics : Social Work and Political Responsability, Maryland, National Association of Social Workers, $1982: 1-10$, citations traduites par Gérald Doré.

CAstells, M., Luttes urbaines, Paris, Maspéro, 1975.

Chancel, J., "En deçà, au-delà du Politique... les portes étroites ", Autrement, $n^{\circ} 29,1981$.

Collin, J.P. et J. GODBOUT, Les organismes populaires en milieu urbain : contrepouvoir ou nouvelles pratiques professionnelles? Montréal, I.N.R.S.Urbanisation, 1977.

DORÉ, G., La politique sociale comme pratique de classes, thèse de doctorat en sociologie, Québec, Université Laval, 1978.

DORÉ, G. et R. MAYER, "L'idéologie du réaménagement urbain à Québec ", dans AA. VV. Une ville à vendre, Montréal, Saint-Martin, 1981 : 353-504.

DORÉ, G. et D. PLAMONDON, "Les pratiques urbaines d'opposition à Québec ", Revue internationale $d^{\prime}$ action communautaire, vol. 4, $n^{\circ} 44,1980: 120-128$.

FORTIN, D., "La recherche-action à caractère militant : le cas du GRAP ", Service Social, vol. 34, $\mathrm{n}^{\text {os }}$ 2-3, $1985:$ 269-293.

ForTIN, D., Riches contre pauvres, Québec, Éditions autogestionnaires, 1988.

Fortin, D. et M. ROLAND, Histoire des luttes de protection des consommateurs au Québec (1962-1978), Québec, Groupe de recherche en action populaire, cahier 1, 1981. 
Hamel, P., Logement et luttes urbaines à Montréal (1963-1976), Faculté de I'aménagement, Université de Montréal, 1983.

LeBOeUf, L., "Vaincre la peur et les préjugés. La lutte contre les bouboumacoutes ", dans AA. VV., Pratiques de conscientisation 2, Québec, Collectif québécois d'édition populaire, $1987:$ 109-158.

Macciocchi, M.-A., Pour Gramsci, Paris, Seuil, Coll. "Points », 1974.

Mahaffey, M. et J.W. Hanks (sous la direction de), Practical Politics : Social Work and Political Responsability, Maryland, National Association of Social Workers, 1982, citations traduites par Gérald Doré.

Marshall, G. et C.T. Strandberg, "Politics and Social Work. The Professional Interface ", The Social Worker/Le travailleur social, vol. 56, n 3, 1988 : 112-116, citations traduites par Gérald Doré.

McGraw, D., Le développement des groupes populaires à Montréal, Montréal, Saint-Martin, 1978.

MERTON, R.K., Éléments de théorie et de méthode sociologique, Paris, Plon, 1965.

PANITCH, L., "The role and nature of the Canadian state", dans PANITCH, L. (sous la direction de), The Canadian State. Political Economy and Political Power, Toronto, University of Toronto Press, 1977 : 3-27.

PATTI, R.J., "Political Action », dans Supplement to the Encyclopedia of Social Work 1983-84, Maryland, National Association of Social Workers, 1983, citations traduites par Gérald Doré.

Pelletier, M. et Y. Vaillancourt, Les politiques sociales et les travailleurs, Montréal, à compte d'auteurs, 1974-1978.

PIVen, F.F. et R.A. Cloward, Regulating the Poor: the Functions of Public Welfare, New York, Pantheon Books, 1971.

Pizzorno, A., I soggetti del pluralismo. Classi. Partiti. Sindacati, Bologna, II Mulino, 1980, citations traduites par Gérald Doré.

PoIRIER, R., Qui a volé la rue principale ? Montréal, Éditions Départ, 1986.

RenÉ, J.-F. et J. PANET-RAYMOND, Faut-il brûler les pancartes? Montréal, Association coopérative d'économie familiale, 1984.

RoberGe, C., Lutte des locataires des Appartements Saint-Pie X pour l'implantation de services dans leur complexe d'habitation H.L.M., 1971-1977, thèse de maîtrise en service social, Québec, Université Laval, 1981.

ROBERT, L. et P. RACICOT, "Le Comité de citoyens de l'aire 10 : une contrepratique politique? " dans AA. VV., Une ville à vendre, Montréal, SaintMartin, 1981 : 319-339.

TOURAINE, A., "La crise de la représentation politique ", Sociologie et sociétés, vol. 15, no 1, $1983: 131-140$.

VAILLANCOURT, Y., Le P.Q. et le social : éléments de bilan des politiques sociales du gouvernement du Parti Québécois, 1976-1982, Montréal, Éditions Socialisme et indépendance, 1983.

VaillanCOURT, Y., L'évolution des politiques sociales au Québec, 1940-1960, Montréal, Les Presses de l'Université de Montréal, 1988.

VALOIS, J. et P. PAQUET, Les groupes populaires dans la structure du pouvoir, Montréal, Institut canadien d'éducation des adultes, 1974.

Ventelou, D., La lutte des assistés sociaux de Montréal contre la taxe d'eau, Québec, Groupe de recherche en action populaire, cahier 8, 1983.

WELCH, D., Trois mobilisations populaires dans un quartier prolétaire, Montréal, Agence de presse libre du Québec, 1975. 